Bulletin of the Institute of Mathematics

Academia Sinica (New Series)

Vol. 12 (2017), No. 2, pp. 103-124

DOI: 10.21915 /BIMAS.2017201

\title{
EXACT WEAK LAWS AND ONE SIDED STRONG LAWS
}

\author{
ANDRÉ ADLER
}

Department of Applied Mathematics, Illinois Institute of Technology, Chicago, Illinois, 60616, USA. E-mail: adler@iit.edu

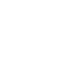

\begin{abstract}
This paper explores Exact Weak Laws and their almost sure counterparts. First we show that the weighted sums of our infinite mean random variables can be balanced by a carefully selected sequence, i.e., that the ratio converges to one in probability. Then it is shown that the upper limit is almost surely infinity, while the lower limit is one.
\end{abstract}

\section{Introduction}

An exact limit theorem is where the numerator and denominator in some sense approaches one. That means that the sum of the random variables in the numerator are balanced appropriately by the constants in the denominator. The sum in the numerator can be considered the winnings of some game while the denominator would be the cumulative entrance fee after those $n$ games have been played. The house and the gambler would only play this game if it was fair in some sense.

In this paper we extend the work done in [2] and [5]. Here we generalize the distribution used in both papers to $P\left\{X_{j}>x\right\}=\frac{\log ^{\alpha}(x+j)}{x+j}$. We use the classic slowly varying function logarithm and since we want our random variables to have infinite expectations, we set $\alpha \geq-1$. Besides establishing the Weak Laws (Section 2) we also obtain the precise almost sure bounds (Section 3). As suggested in [2] and done in [5], it would be nice to extend this work for a more general sequence than just $j$. We would like to do both, we would like to obtain limit theorems when $P\left\{X_{j}>x\right\}=\frac{L\left(x+c_{j}\right)}{x+c_{j}}$

Received September 11, 2016.

AMS Subject Classification: 60F05, 60F15.

Key words and phrases: Almost sure convergence, weak law of large numbers, exact laws, one sided strong laws, slow variation. 
for any sequence $c_{j}$ and any slowly varying function $L(x)$. But that is very complicated, especially determining which random variables do not have a finite mean. So we will stay with $c_{j}=j$, but look at various powers of logarithm.

Since the random variables have $1-F_{X_{j}}(x)=\frac{\log ^{\alpha}(x+j)}{x+j}$ where $x>0$ and $\alpha>-1$, we set $P\left\{X_{j}=0\right\}=1-\frac{\log ^{\alpha}(j)}{j}$ and by differentiating $F_{X_{j}}(x)$ we obtain

$$
f_{X_{j}}(x)=\frac{\log ^{\alpha}(x+j)-\alpha \log ^{\alpha-1}(x+j)}{(x+j)^{2}} .
$$

In terms of notation we use $\lg x=\log x$ with the base $e$, but whenever we have $\log 1$ in a denominator we will just set that equal to one, so we won't be dividing by zero. Hence $\lg x=\ln x$, except $\lg 1=1$. We set $\lg _{2} x=\lg (\lg x)$, $\lg _{3} x=\lg \left(\lg _{2} x\right)$ and $\lg _{4} x=\lg \left(\lg _{3} x\right)$, so the $i$ in $\lg _{i} x$ is not the base, it is the iteration of the logarithm. Many times in the proofs we will pull the logarithm out from an integral, since it is a slowly varying function, see [4], pages 279-284. Finally, note that the constant $C$ will be used as a generic bound that is not necessarily the same in each appearance.

\section{Exact Weak Laws}

We first look at $\alpha>-1$, then we explore what happens when $\alpha$ equals minus one.

Theorem 1. If $P\left\{X_{j}>x\right\}=\frac{\lg ^{\alpha}(x+j)}{x+j}$ where $\alpha>-1$, then

$$
\frac{\sum_{j=1}^{n} \frac{1}{j} X_{j}}{\lg ^{\alpha+1}(n) \lg _{2}(n)} \stackrel{P}{\rightarrow} 1 .
$$

Proof. We will establish all three parts of the Degenerate Convergence Criterion, see [3], page 356. Let $a_{j}=1 / j$ and $b_{n}=\lg ^{\alpha+1}(n) \lg _{2}(n)$. Even though all the terms are quite similar, we will see that all of them go to zero except for one. And that one is only one of four in our truncated mean

$$
\sum_{j=1}^{n} \frac{a_{j}}{b_{n}} E X_{j} I\left(a_{j} X_{j} \leq b_{n}\right)
$$




$$
\begin{aligned}
= & \frac{1}{b_{n}} \sum_{j=1}^{n} \frac{1}{j} \int_{0}^{b_{n} j} x\left[\frac{\lg ^{\alpha}(x+j)-\alpha \lg ^{\alpha-1}(x+j)}{(x+j)^{2}}\right] d x \\
= & \frac{1}{b_{n}} \sum_{j=1}^{n} \frac{1}{j} \int_{j}^{\left(b_{n}+1\right) j}(u-j)\left[\frac{\lg ^{\alpha}(u)-\alpha \lg ^{\alpha-1}(u)}{u^{2}}\right] d u \\
= & \frac{1}{b_{n}} \sum_{j=1}^{n} \frac{1}{j} \int_{j}^{\left(b_{n}+1\right) j}\left[\frac{\lg ^{\alpha}(u)}{u}-\frac{j \lg ^{\alpha}(u)}{u^{2}}-\frac{\alpha \lg ^{\alpha-1}(u)}{u}+\frac{\alpha j \lg ^{\alpha-1}(u)}{u^{2}}\right] d u \\
= & \frac{1}{b_{n}} \sum_{j=1}^{n} \frac{1}{j} \int_{j}^{\left(b_{n}+1\right) j} \frac{\lg ^{\alpha}(u) d u}{u}-\frac{1}{b_{n}} \sum_{j=1}^{n} \int_{j}^{\left(b_{n}+1\right) j} \frac{\lg ^{\alpha}(u) d u}{u^{2}} \\
& -\frac{\alpha}{b_{n}} \sum_{j=1}^{n} \frac{1}{j} \int_{j}^{\left(b_{n}+1\right) j} \frac{\lg ^{\alpha-1}(u) d u}{u}+\frac{\alpha}{b_{n}} \sum_{j=1}^{n} \int_{j}^{\left(b_{n}+1\right) j} \frac{\lg ^{\alpha-1}(u) d u}{u^{2}} \\
= & I_{1}+I_{2}+I_{3}+I_{4} .
\end{aligned}
$$

Letting $w=\lg u$, the first term becomes

$$
\begin{aligned}
I_{1} & =\frac{1}{b_{n}} \sum_{j=1}^{n} \frac{1}{j} \int_{j}^{\left(b_{n}+1\right) j} \frac{\lg ^{\alpha}(u) d u}{u} \\
& =\frac{1}{b_{n}} \sum_{j=1}^{n} \frac{1}{j} \int_{\lg (j)}^{\lg \left[\left(b_{n}+1\right) j\right]} w^{\alpha} d w \\
& =\frac{1}{(\alpha+1) b_{n}} \sum_{j=1}^{n} \frac{1}{j}\left[\lg ^{\alpha+1}\left(\left(b_{n}+1\right) j\right)-\lg ^{\alpha+1}(j)\right] \\
& =\frac{1}{(\alpha+1) b_{n}} \sum_{j=1}^{n} \frac{\lg ^{\alpha+1}(j)}{j}\left[\left(\frac{\lg \left(b_{n}+1\right)+\lg (j)}{\lg (j)}\right)^{\alpha+1}-1\right] \\
& =\frac{1}{(\alpha+1) b_{n}} \sum_{j=1}^{n} \frac{\lg ^{\alpha+1}(j)}{j}\left[\left(1+\frac{\lg \left(b_{n}+1\right)}{\lg (j)}\right)^{\alpha+1}-1\right]
\end{aligned}
$$

The Taylor Series for $(1+x)^{\alpha+1}$ is

$$
(1+x)^{\alpha+1}=1+(\alpha+1) x+\frac{(\alpha+1) \alpha x^{2}}{2}+\frac{(\alpha+1) \alpha(\alpha-1) x^{3}}{6}+\cdots
$$

which is the usual Binomial Theorem if $\alpha+1$ is an integer, meaning it is a finite sum. Otherwise, it becomes an alternating series and the error is less than the ensuing term, which is just as good, since $x=\lg \left(b_{n}+1\right) / \lg (j)$ 
is small as long as $j$ exceeds some value say $J_{0}$. Thus the main term is essentially

$$
\begin{aligned}
& \frac{1}{(\alpha+1) b_{n}} \sum_{j=1}^{n} \frac{\lg ^{\alpha+1}(j)}{j}\left[\frac{(\alpha+1) \lg \left(b_{n}+1\right)}{\lg (j)}\right] \\
& \quad \sim \frac{\lg \left(b_{n}\right)}{b_{n}} \sum_{j=1}^{n} \frac{\lg ^{\alpha}(j)}{j} \\
& \sim\left(\frac{\lg \left(b_{n}\right)}{b_{n}}\right)\left(\frac{\lg ^{\alpha+1}(n)}{\alpha+1}\right) \\
& \sim\left(\frac{(\alpha+1) \lg _{2}(n)}{\lg ^{\alpha+1}(n) \lg _{2}(n)}\right)\left(\frac{\lg ^{\alpha+1}(n)}{\alpha+1}\right)=1 .
\end{aligned}
$$

As for any of the higher powers in this expansion, say $p \geq 2$, they are bounded above by

$$
\frac{C}{b_{n}} \sum_{j=1}^{n} \frac{\lg ^{\alpha+1}(j)}{j}\left(\frac{\lg \left(b_{n}+1\right)}{\lg (j)}\right)^{p} \leq \frac{C \lg ^{p}\left(b_{n}\right)}{b_{n}} \sum_{j=1}^{n} \frac{\lg ^{\alpha-p+1}(j)}{j}
$$

And by examining the the three cases we get, if $\alpha-p+1<-1$

$$
\frac{C \lg ^{p}\left(b_{n}\right)}{b_{n}} \sum_{j=1}^{n} \frac{\lg ^{\alpha-p+1}(j)}{j} \leq \frac{C \lg ^{p}\left(b_{n}\right)}{b_{n}} \rightarrow 0
$$

and if $\alpha-p+1=-1$, it is bounded above by

$$
\begin{aligned}
\frac{C \lg ^{p}\left(b_{n}\right)}{b_{n}} \sum_{j=1}^{n} \frac{\lg ^{\alpha-p+1}(j)}{j} & \leq \frac{C \lg ^{p}\left(b_{n}\right) \lg _{2}(n)}{b_{n}} \leq \frac{C \lg _{2}^{p+1}(n)}{\lg ^{\alpha+1}(n) \lg _{2}(n)} \\
& =\frac{C \lg _{2}^{p}(n)}{\lg ^{p-1}(n)} \rightarrow 0
\end{aligned}
$$

and finally if $\alpha-p+1>-1$, then this term is bounded above by

$$
\begin{aligned}
& \frac{C \lg ^{p}\left(b_{n}\right)}{b_{n}} \sum_{j=1}^{n} \frac{\lg ^{\alpha-p+1}(j)}{j} \\
& \leq \frac{C \lg ^{p}\left(b_{n}\right) \lg ^{\alpha-p+2}(n)}{b_{n}} \leq \frac{C \lg _{2}^{p}(n) \lg ^{\alpha-p+2}(n)}{\lg ^{\alpha+1}(n) \lg _{2}(n)}=\frac{C \lg _{2}^{p-1}(n)}{\lg ^{p-1}(n)} \rightarrow 0 .
\end{aligned}
$$

This shows how delicate the selection of $b_{n}$ is. Now we need to examine the 
other three terms in our truncated mean. Those and the other two terms in the Degenerate Convergence Theorem all go to zero. The second term in the truncated mean, by using slow variation, is bounded above by

$$
\begin{aligned}
\left|I_{2}\right| & =\left|\frac{-1}{b_{n}} \sum_{j=1}^{n} \int_{j}^{\left(b_{n}+1\right) j} \frac{\lg ^{\alpha}(u) d u}{u^{2}}\right| \leq \frac{1}{b_{n}} \sum_{j=1}^{n} \int_{j}^{\infty} \frac{\lg ^{\alpha}(u) d u}{u^{2}} \\
& \leq \frac{C}{b_{n}} \sum_{j=1}^{n} \frac{\lg ^{\alpha}(j)}{j} \leq \frac{C \lg ^{\alpha+1}(n)}{b_{n}}=\frac{C}{\lg _{2}(n)} \rightarrow 0 .
\end{aligned}
$$

The third term is similar to the first, but with one less power of logarithm, it is bounded above by

$$
\begin{aligned}
\left|I_{3}\right| & =\left|\frac{-\alpha}{b_{n}} \sum_{j=1}^{n} \frac{1}{j} \int_{j}^{\left(b_{n}+1\right) j} \frac{\lg ^{\alpha-1}(u) d u}{u}\right| \\
& \leq \frac{C}{b_{n}} \sum_{j=1}^{n} \frac{1}{j} \int_{j}^{\left(b_{n}+1\right) j} \frac{\lg ^{\alpha-1}(u) d u}{u} \\
& \leq \frac{C}{b_{n}} \sum_{j=1}^{n} \frac{1}{j} \int_{\lg (j)}^{\lg \left[\left(b_{n}+1\right) j\right]} w^{\alpha-1} d w \\
& \leq \frac{C}{b_{n}} \sum_{j=1}^{n} \frac{1}{j}\left[\lg \alpha\left(\left(b_{n}+1\right) j\right)-\lg ^{\alpha}(j)\right] \\
& =\frac{C}{b_{n}} \sum_{j=1}^{n} \frac{\lg ^{\alpha}(j)}{j}\left[\left(\frac{\lg \left(b_{n}+1\right)+\lg (j)}{\lg (j)}\right)^{\alpha}-1\right] \\
& =\frac{C}{b_{n}} \sum_{j=1}^{n} \frac{\lg ^{\alpha}(j)}{j}\left[\left(1+\frac{\lg \left(b_{n}+1\right)}{\lg (j)}\right)^{\alpha}-1\right] .
\end{aligned}
$$

Using the Binomial expansion once again, we have for the first term

$$
\begin{aligned}
\frac{C}{b_{n}} \sum_{j=1}^{n}\left(\frac{\lg ^{\alpha}(j)}{j}\right)\left(\frac{\alpha \lg \left(b_{n}+1\right)}{\lg (j)}\right) & \leq \frac{C \lg \left(b_{n}\right)}{b_{n}} \sum_{j=1}^{n} \frac{\lg ^{\alpha-1}(j)}{j} \\
& \leq \frac{C \lg \left(b_{n}\right) \lg ^{\alpha}(n)}{b_{n}} \leq \frac{C}{\lg n} \rightarrow 0
\end{aligned}
$$

Likewise, the ensuing powers in the expansion make those terms go to zero 
as well. Finally, the fourth term in our mean is

$$
\begin{aligned}
I_{4} & =\frac{\alpha}{b_{n}} \sum_{j=1}^{n} \int_{j}^{\left(b_{n}+1\right) j} \frac{\lg ^{\alpha-1}(u) d u}{u^{2}} \leq \frac{C}{b_{n}} \sum_{j=1}^{n} \int_{j}^{\infty} \frac{\lg ^{\alpha-1}(u) d u}{u^{2}} \\
& \leq \frac{C}{b_{n}} \sum_{j=1}^{n} \frac{\lg ^{\alpha-1}(j)}{j} \leq \frac{C \lg ^{\alpha}(n)}{b_{n}}=\frac{C}{\lg (n) \lg _{2}(n)} \rightarrow 0 .
\end{aligned}
$$

Note that, we just assumed that $\alpha>0$, but if $-1<\alpha \leq 0$, these terms also vanishes as $\mathrm{n}$ goes to infinity.

Next we look at the second moment term in the Degenerate Convergence Theorem

$$
\begin{aligned}
& \frac{1}{b_{n}^{2}} \sum_{j=1}^{n} a_{j}^{2} E X_{j}^{2} I\left(a_{j} X_{j} \leq b_{n}\right) \\
& =\frac{1}{b_{n}^{2}} \sum_{j=1}^{n} \frac{1}{j^{2}} \int_{0}^{b_{n} j} x^{2}\left[\frac{\lg ^{\alpha}(x+j)-\alpha \lg ^{\alpha-1}(x+j)}{(x+j)^{2}}\right] d x \\
& \leq \frac{C}{b_{n}^{2}} \sum_{j=1}^{n} \frac{1}{j^{2}} \int_{0}^{b_{n} j} \frac{x^{2} \lg ^{\alpha}(x+j) d x}{(x+j)^{2}} \\
& \leq \frac{C}{b_{n}^{2}} \sum_{j=1}^{n} \frac{1}{j^{2}} \int_{0}^{b_{n} j} \lg ^{\alpha}(x+j) d x \\
& =\frac{C}{b_{n}^{2}} \sum_{j=1}^{n} \frac{1}{j^{2}} \int_{j}^{\left(b_{n}+1\right) j} \lg ^{\alpha}(u) d u .
\end{aligned}
$$

If $\alpha=0$, which was already established in [2], then this term is

$$
\frac{C}{b_{n}^{2}} \sum_{j=1}^{n} \frac{1}{j^{2}}\left[\left(b_{n}+1\right) j-j\right]=\frac{C}{b_{n}} \sum_{j=1}^{n} \frac{1}{j} \leq \frac{C}{\lg _{2}(n)} \rightarrow 0 .
$$

And if $-1<\alpha<0$, then this term is

$$
\frac{C}{b_{n}^{2}} \sum_{j=1}^{n} \frac{1}{j^{2}} \int_{j}^{\left(b_{n}+1\right) j} \lg ^{\alpha}(u) d u \leq \frac{C}{b_{n}} \sum_{j=1}^{n} \frac{\lg ^{\alpha}(j)}{j} \leq \frac{C \lg ^{\alpha+1}(n)}{b_{n}}=\frac{C}{\lg _{2}(n)} \rightarrow 0
$$


Finally, if $\alpha>0$, then this term is

$$
\begin{aligned}
\frac{C}{b_{n}^{2}} \sum_{j=1}^{n} \frac{1}{j^{2}} \int_{j}^{\left(b_{n}+1\right) j} \lg ^{\alpha}(u) d u & \leq \frac{C}{b_{n}^{2}} \sum_{j=1}^{n} \frac{1}{j^{2}} \lg ^{\alpha}\left(\left(b_{n}+1\right) j\right) \int_{j}^{\left(b_{n}+1\right) j} d u \\
& =\frac{C}{b_{n}^{2}} \sum_{j=1}^{n} \frac{1}{j^{2}} \lg ^{\alpha}\left(\left(b_{n}+1\right) j\right)\left[\left(b_{n}+1\right) j-j\right] \\
& =\frac{C}{b_{n}^{2}} \sum_{j=1}^{n} \frac{1}{j^{2}} \lg ^{\alpha}\left(\left(b_{n}+1\right) j\right)\left[b_{n} j\right] \\
& =\frac{C}{b_{n}} \sum_{j=1}^{n} \frac{1}{j} \lg ^{\alpha}\left(\left(b_{n}+1\right) j\right) \\
& =\frac{C}{b_{n}} \sum_{j=1}^{n} \frac{1}{j}\left(\lg \left(b_{n}+1\right)+\lg j\right)^{\alpha} \\
& =\frac{C}{b_{n}} \sum_{j=1}^{n} \frac{\lg ^{\alpha}(j)}{j}\left(\frac{\lg \left(b_{n}+1\right)}{\lg (j)}+1\right)^{\alpha}
\end{aligned}
$$

Once again using the expansion $(1+x)^{\alpha}=1+\alpha x+\alpha(\alpha-1) x^{2} / 2 \cdots$ which is either finite or eventually an alternating series, we see that this limit is zero. The first term is

$$
\frac{C}{b_{n}} \sum_{j=1}^{n} \frac{\lg ^{\alpha}(j)}{j}(1) \leq \frac{C \lg ^{\alpha+1}(n)}{b_{n}}=\frac{C}{\lg _{2}(n)} \rightarrow 0 .
$$

The next term is $\alpha x$, where $x=\lg \left(b_{n}+1\right) / \lg (j)$, which is small for most values of $j$. We could split the sum into two parts, where one sum is $\sum_{j=1}^{J_{0}}$ and the second sum is $\sum_{j=J_{0}+1}^{n}$ where $x<1$, when $j$ exceeds $J_{0}$. The first sum would go to zero as $n$ went to infinity since $b_{n} \rightarrow \infty$. Thus the second term is in our series expansion of $(1+x)^{\alpha}$ is

$$
\begin{aligned}
\frac{C}{b_{n}} \sum_{j=1}^{n} \frac{\lg ^{\alpha}(j)}{j}\left(\frac{\alpha \lg \left(b_{n}+1\right)}{\lg (j)}\right) & \leq \frac{C \lg \left(b_{n}\right)}{b_{n}} \sum_{j=1}^{n} \frac{\lg ^{\alpha-1}(j)}{j} \\
& \leq \frac{C \lg _{2}(n) \lg ^{\alpha}(n)}{\lg ^{\alpha+1}(n) \lg _{2}(n)}=\frac{C}{\lg n} \rightarrow 0 .
\end{aligned}
$$

Now the rest of the binomial series has powers $x^{p}$ for $p \geq 2$. Either this series terminates or eventually alternates. In either case for the term $x^{p}$, we 
have

$$
\begin{aligned}
\frac{C}{b_{n}} \sum_{j=1}^{n} \frac{\lg ^{\alpha}(j)}{j}\left(\frac{\lg \left(b_{n}+1\right)}{\lg (j)}\right)^{p} & \leq \frac{C \lg ^{p}\left(b_{n}\right)}{b_{n}} \sum_{j=1}^{n} \frac{\lg ^{\alpha-p}(j)}{j} \\
& \leq \frac{C \lg _{2}^{p}(n) \lg ^{\alpha-p+1}(n)}{\lg ^{\alpha+1}(n) \lg _{2}(n)}=\frac{C \lg _{2}^{p-1}(n)}{\lg ^{p}(n)} \rightarrow 0
\end{aligned}
$$

where we are assuming that $\alpha-p+1>0$, but we easily show that all of these terms go to zero in every setting. Thus the variance term does vanish as $n$ goes to infinity.

The last limit in the Degenerate Convergence Theorem are the tail probabilities, but that limit is zero just like the last one, since for all $\epsilon>0$

$$
\begin{aligned}
\sum_{j=1}^{n} P\left\{X_{j}>\epsilon b_{n} j\right\} & =\sum_{j=1}^{n} \frac{\left(\lg \left(\epsilon b_{n} j+j\right)\right)^{\alpha}}{\epsilon b_{n} j+j} \\
& =\frac{1}{\epsilon b_{n}+1} \sum_{j=1}^{n} \frac{\left(\lg \left(\epsilon b_{n}+1\right)+\lg (j)\right)^{\alpha}}{j} \\
& \leq \frac{C}{b_{n}} \sum_{j=1}^{n} \frac{\left(\lg \left(\epsilon b_{n}+1\right)+\lg (j)\right)^{\alpha}}{j}
\end{aligned}
$$

If $-1<\alpha \leq 0$, then this is bounded above by

$$
\frac{C}{b_{n}} \sum_{j=1}^{n} \frac{\lg ^{\alpha}(j)}{j} \leq \frac{C \lg ^{\alpha+1}(n)}{b_{n}}=\frac{C}{\lg _{2}(n)} \rightarrow 0 .
$$

However, if $\alpha>0$, this term becomes

$$
\frac{C}{b_{n}} \sum_{j=1}^{n} \frac{\lg ^{\alpha}(j)}{j}\left(\frac{\lg \left(\epsilon b_{n}+1\right)}{\lg (j)}+1\right)^{\alpha} .
$$

Again using $(1+x)^{\alpha}=1+\alpha x+\alpha(\alpha-1) x^{2} / 2 \cdots$, which is either a finite sum or an alternating series, we have for the first term

$$
\frac{C}{b_{n}} \sum_{j=1}^{n} \frac{\lg ^{\alpha}(j)}{j}(1) \leq \frac{C \lg ^{\alpha+1}(n)}{b_{n}}=\frac{C}{\lg _{2}(n)} \rightarrow 0 .
$$


Next, observing the $\alpha x$ term, which is

$$
\begin{aligned}
\frac{C}{b_{n}} \sum_{j=1}^{n} \frac{\lg ^{\alpha}(j)}{j}\left(\frac{\alpha \lg \left(\epsilon b_{n}+1\right)}{\lg (j)}\right) & \leq \frac{C \lg \left(\epsilon b_{n}+1\right)}{b_{n}} \sum_{j=1}^{n} \frac{\lg ^{\alpha-1}(j)}{j} \\
& \leq \frac{C \lg _{2}(n) \lg ^{\alpha}(n)}{\lg ^{\alpha+1}(n) \lg _{2}(n)}=\frac{C}{\lg n} \rightarrow 0 .
\end{aligned}
$$

The value of $x=\lg \left(\epsilon b_{n}+1\right) / \lg (j)$, is small for most values of $j$ in our series expansion of $(1+x)^{\alpha}$. We can make this rigorous by splitting the sum into two parts, where one sum is $\sum_{j=1}^{J_{0}}$ and the second sum is $\sum_{j=J_{0}+1}^{n}$ where $x<1$, when $j$ exceeds $J_{0}$, but that isn't necessary. This series either terminates, when $\alpha$ is an integer or it eventually alternates, so looking at the $x^{p}$ term with $\mathrm{p}$ an integer greater than one, we have

$$
\frac{C}{b_{n}} \sum_{j=1}^{n} \frac{\lg ^{\alpha}(j)}{j}\left(\frac{\lg \left(\epsilon b_{n}+1\right)}{\lg (j)}\right)^{p} \leq \frac{C \lg ^{p}\left(b_{n}\right)}{b_{n}} \sum_{j=1}^{n} \frac{\lg ^{\alpha-p}(j)}{j} .
$$

If $\alpha-p<-1$, this is bounded above by

$$
\frac{C \lg ^{p}\left(b_{n}\right)}{b_{n}} \leq \frac{C \lg _{2}^{p}(n)}{\lg ^{\alpha+1}(n) \lg _{2}(n)}=\frac{C \lg _{2}^{p-1}(n)}{\lg ^{\alpha+1}(n)} \rightarrow 0 .
$$

If $\alpha-p=-1$, this is bounded above by

$$
\frac{C \lg _{2}^{p+1}(n)}{\lg ^{\alpha+1}(n) \lg _{2}(n)}=\frac{C \lg _{2}^{p}(n)}{\lg ^{p}(n)} \rightarrow 0 \text {. }
$$

Finally, if $\alpha-p$ exceeds minus one, an upper bound is

$$
\frac{C \lg _{2}^{p}(n) \lg ^{\alpha-p+1}(n)}{\lg ^{\alpha+1}(n) \lg _{2}(n)}=\frac{C \lg _{2}^{p-1}(n)}{\lg ^{p}(n)} \rightarrow 0
$$

completing this proof.

We set the parameter $\alpha$ larger than minus one, so that these random variables have infinite expectation. If the expectation was finite, then the classic Laws of Large Numbers would apply. Next, we examine what happens when $\alpha=-1$. In keeping with the spirit of non-integrable random variables, we now assume $P\left\{X_{j}>x\right\}=\frac{1}{(x+j) \lg (x+j)}$. Thus $P\left\{X_{j}=0\right\}=1-\frac{1}{j \lg (j)}$ 
and by differentiating $F_{X_{j}}(x)=1-P\left\{X_{j}>x\right\}$ we obtain

$$
f_{X_{j}}(x)=\frac{\lg (x+j)+1}{(x+j)^{2} \lg ^{2}(x+j)} .
$$

Theorem 2. If $P\left\{X_{j}>x\right\}=\frac{1}{(x+j) \lg (x+j)}$, then

$$
\frac{\sum_{j=1}^{n} \frac{1}{j} X_{j}}{\lg _{2}(n) \lg _{3}(n)} \stackrel{P}{\rightarrow} 1 .
$$

Proof. We start once again by examining the truncated mean, which shows that our selection of $b_{n}=\lg _{2}(n) \lg _{3}(n)$ is the correct choice

$$
\begin{aligned}
\sum_{j=1}^{n} \frac{a_{j}}{b_{n}} E X_{j} I\left(a_{j} X_{j} \leq b_{n}\right) \\
=\frac{1}{b_{n}} \sum_{j=1}^{n} \frac{1}{j} \int_{0}^{b_{n} j} x\left[\frac{\lg (x+j)+1}{(x+j)^{2} \lg ^{2}(x+j)}\right] d x \\
=\frac{1}{b_{n}} \sum_{j=1}^{n} \frac{1}{j} \int_{j}^{\left(b_{n}+1\right) j}(u-j)\left[\frac{\lg (u)+1}{u^{2} \lg ^{2}(u)}\right] d u \\
=\frac{1}{b_{n}} \sum_{j=1}^{n} \frac{1}{j} \int_{j}^{\left(b_{n}+1\right) j} \frac{d u}{u \lg (u)}-\frac{1}{b_{n}} \sum_{j=1}^{n} \int_{j}^{\left(b_{n}+1\right) j} \frac{d u}{u^{2} \lg (u)} \\
\quad+\frac{1}{b_{n}} \sum_{j=1}^{n} \frac{1}{j} \int_{j}^{\left(b_{n}+1\right) j} \frac{d u}{u \lg ^{2}(u)}-\frac{1}{b_{n}} \sum_{j=1}^{n} \int_{j}^{\left(b_{n}+1\right) j} \frac{d u}{u^{2} \lg ^{2}(u)} \\
=I_{1}+I_{2}+I_{3}+I_{4} .
\end{aligned}
$$

Letting $w=\lg (u)$, the first term becomes

$$
\begin{aligned}
I_{1} & =\frac{1}{b_{n}} \sum_{j=1}^{n} \frac{1}{j} \int_{j}^{\left(b_{n}+1\right) j} \frac{d u}{u \lg u}=\frac{1}{b_{n}} \sum_{j=1}^{n} \frac{1}{j} \int_{\lg j}^{\lg \left[\left(b_{n}+1\right) j\right]} \frac{d w}{w} \\
& =\frac{1}{b_{n}} \sum_{j=1}^{n} \frac{1}{j}\left[\lg _{2}\left(\left(b_{n}+1\right) j\right)-\lg _{2}(j)\right]=\frac{1}{b_{n}} \sum_{j=1}^{n} \frac{1}{j} \lg \left[\frac{\lg \left(b_{n}+1\right)+\lg (j)}{\lg (j)}\right] \\
& =\frac{1}{b_{n}} \sum_{j=1}^{n} \frac{1}{j} \lg \left[\frac{\lg \left(b_{n}+1\right)}{\lg (j)}+1\right] .
\end{aligned}
$$

We use the series expansion of $\lg (x+1)=x-x^{2} / 2+x^{3} / 3+\cdots$ with $x=\lg \left(b_{n}+1\right) / \lg (j)$, which is small if $j$ exceeds some value, say $J_{0}$. This is 
an alternating series and the only significant term is the $x$ term. Plugging in $x$ into $I_{1}$ we have

$$
\frac{1}{b_{n}} \sum_{j=1}^{n} \frac{\lg \left(b_{n}+1\right)}{j \lg (j)} \sim \frac{\lg \left(b_{n}\right)}{b_{n}} \sum_{j=1}^{n} \frac{1}{j \lg (j)} \sim \frac{\lg _{3}(n) \lg _{2}(n)}{b_{n}}=1 .
$$

Any other term, $x^{p}$, with $p \geq 2$ is

$$
\frac{1}{b_{n}} \sum_{j=1}^{n} \frac{\lg ^{p}\left(b_{n}+1\right)}{j \lg ^{p}(j)} \leq \frac{C \lg ^{p}\left(b_{n}\right)}{b_{n}} \leq \frac{\lg _{3}^{p-1}(n)}{\lg _{2}(n)} \rightarrow 0
$$

since $\lg \left(b_{n}\right)=\lg _{3}(n)+\lg _{4}(n) \sim \lg _{3}(n)$. That is the only nonzero limit, since

$$
\begin{aligned}
\left|I_{2}\right| & =\left|\frac{-1}{b_{n}} \sum_{j=1}^{n} \int_{j}^{\left(b_{n}+1\right) j} \frac{d u}{u^{2} \lg (u)}\right| \leq \frac{1}{b_{n}} \sum_{j=1}^{n} \int_{j}^{\infty} \frac{d u}{u^{2} \lg (u)} \\
& \leq \frac{C}{b_{n}} \sum_{j=1}^{n} \frac{1}{j \lg (j)} \leq \frac{C \lg _{2}(n)}{b_{n}}=\frac{C}{\lg _{3}(n)} \rightarrow 0
\end{aligned}
$$

and

$$
\begin{aligned}
I_{3} & =\frac{1}{b_{n}} \sum_{j=1}^{n} \frac{1}{j} \int_{j}^{\left(b_{n}+1\right) j} \frac{d u}{u \lg ^{2}(u)} \\
& \leq \frac{1}{b_{n}} \sum_{j=1}^{n} \frac{1}{j} \int_{j}^{\infty} \frac{d u}{u \lg ^{2}(u)} \\
& \leq \frac{C}{b_{n}} \sum_{j=1}^{n} \frac{1}{j \lg (j)} \leq \frac{C \lg _{2}(n)}{b_{n}}=\frac{C}{\lg _{3}(n)} \rightarrow 0
\end{aligned}
$$

and finally

$$
\begin{aligned}
\left|I_{4}\right| & =\left|\frac{-1}{b_{n}} \sum_{j=1}^{n} \int_{j}^{\left(b_{n}+1\right) j} \frac{d u}{u^{2} \lg ^{2}(u)}\right| \leq \frac{1}{b_{n}} \sum_{j=1}^{n} \int_{j}^{\infty} \frac{d u}{u^{2} \lg ^{2}(u)} \\
& \leq \frac{C}{b_{n}} \sum_{j=1}^{n} \frac{1}{j \lg ^{2}(j)} \leq \frac{C}{b_{n}} \rightarrow 0 .
\end{aligned}
$$

Next, we look at the second moment term in the Degenerate Convergence

Theorem

$$
\frac{1}{b_{n}^{2}} \sum_{j=1}^{n} a_{j}^{2} E X_{j}^{2} I\left(a_{j} X_{j} \leq b_{n}\right)=\frac{1}{b_{n}^{2}} \sum_{j=1}^{n} \frac{1}{j^{2}} \int_{0}^{b_{n} j} x^{2}\left[\frac{\lg (x+j)+1}{(x+j)^{2} \lg ^{2}(x+j)}\right] d x
$$




$$
\begin{aligned}
& =\frac{1}{b_{n}^{2}} \sum_{j=1}^{n} \frac{1}{j^{2}} \int_{j}^{\left(b_{n}+1\right) j} \frac{(u-j)^{2}[\lg (u)+1] d u}{u^{2} \lg ^{2}(u)} \\
& \leq \frac{C}{b_{n}^{2}} \sum_{j=1}^{n} \frac{1}{j^{2}} \int_{j}^{\left(b_{n}+1\right) j} \frac{d u}{\lg (u)} \\
& \leq \frac{C}{b_{n}^{2}} \sum_{j=1}^{n} \frac{1}{j^{2} \lg (j)} \int_{j}^{\left(b_{n}+1\right) j} d u=\frac{C}{b_{n}^{2}} \sum_{j=1}^{n} \frac{b_{n} j}{j^{2} \lg (j)} \\
& =\frac{C}{b_{n}} \sum_{j=1}^{n} \frac{1}{j \lg (j)} \leq \frac{C \lg _{2}(n)}{b_{n}}=\frac{C}{\lg _{3}(n)} \rightarrow 0 .
\end{aligned}
$$

As for the tail probabilities, we have for all $\epsilon>0$

$$
\begin{aligned}
\sum_{j=1}^{n} P\left\{X_{j}>\epsilon b_{n} j\right\} & =\sum_{j=1}^{n} \frac{1}{\left(\epsilon b_{n} j+j\right) \lg \left(\epsilon b_{n} j+j\right)} \\
& =\sum_{j=1}^{n} \frac{1}{\left(\epsilon b_{n}+1\right) j \lg \left[\left(\epsilon b_{n}+1\right) j\right]} \\
& =\frac{1}{\epsilon b_{n}+1} \sum_{j=1}^{n} \frac{1}{j\left[\lg \left(\epsilon b_{n}+1\right)+\lg (j)\right]} \\
& \leq \frac{C}{b_{n}} \sum_{j=1}^{n} \frac{1}{j \lg (j)} \\
& \leq \frac{C \lg _{2}(n)}{b_{n}}=\frac{C}{\lg _{3}(n)} \rightarrow 0 .
\end{aligned}
$$

\section{Almost Sure Results}

The Weak Laws established in the last section will be used to obtain one sided Strong Laws. The easy part was done in both [2] and [5]. By using the technique from [1] we can get an exact lower bound. But the problem is in selecting the right sequence $d_{n}$. Note that the in both proofs we need one series to converge and the other series to diverge where both series differ by just a power of $\lg _{2} n$ and $\lg _{3} n$, respectively.

Theorem 3. If $P\left\{X_{j}>x\right\}=\frac{\lg ^{\alpha}(x+j)}{x+j}$ where $\alpha>-1$, then

$$
\liminf _{n \rightarrow \infty} \frac{\sum_{j=1}^{n} \frac{1}{j} X_{j}}{\lg ^{\alpha+1}(n) \lg _{2}(n)}=1 \quad \text { almost surely }
$$


and

$$
\limsup _{n \rightarrow \infty} \frac{\sum_{j=1}^{n} \frac{1}{j} X_{j}}{\lg ^{\alpha+1}(n) \lg _{2}(n)}=\infty \quad \text { almost surely. }
$$

Proof. Let $a_{j}=1 / j, b_{n}=\lg ^{\alpha+1}(n) \lg _{2}(n), c_{n}=b_{n} / a_{n}=n \lg ^{\alpha+1}(n) \lg _{2}(n)$ and $d_{n}=n \lg ^{\alpha+1}(n)$. The difference between $c_{n}$ and $d_{n}$ is subtle, but that is exactly what helps us achieve our lower limit. The upper limit occurs since for all $M>0$

$$
\begin{aligned}
\sum_{n=1}^{\infty} P\left\{a_{n} X_{n} / b_{n}>M\right\} & =\sum_{n=1}^{\infty} P\left\{X_{n}>M c_{n}\right\} \\
& =\sum_{n=1}^{\infty} \frac{\lg ^{\alpha}\left(M c_{n}+n\right)}{M c_{n}+n} \\
& \geq \sum_{n=1}^{\infty} \frac{\lg ^{\alpha}(n)}{M n \lg ^{\alpha+1}(n) \lg _{2}(n)+n} \\
& \geq C \sum_{n=1}^{\infty} \frac{\lg ^{\alpha}(n)}{n \lg ^{\alpha+1}(n) \lg _{2}(n)} \\
& =C \sum_{n=1}^{\infty} \frac{1}{n \lg (n) \lg _{2}(n)}=\infty
\end{aligned}
$$

when $\alpha \geq 0$. Similarly if $-1<\alpha<0$, then

$$
\begin{aligned}
\sum_{n=1}^{\infty} P\left\{a_{n} X_{n} / b_{n}>M\right\} & =\sum_{n=1}^{\infty} P\left\{X_{n}>M c_{n}\right\} \\
& =\sum_{n=1}^{\infty} \frac{\lg ^{\alpha}\left(M c_{n}+n\right)}{M c_{n}+n} \\
& \geq C \sum_{n=1}^{\infty} \frac{\lg ^{\alpha}\left(c_{n}\right)}{c_{n}} \\
& \geq C \sum_{n=1}^{\infty} \frac{\lg ^{\alpha}(n)}{n \lg ^{\alpha+1}(n) \lg _{2}(n)} \\
& =C \sum_{n=1}^{\infty} \frac{1}{n \lg (n) \lg _{2}(n)}=\infty
\end{aligned}
$$

Thus

$$
\limsup _{n \rightarrow \infty} \frac{a_{n} X_{n}}{b_{n}}=\infty \quad \text { almost surely }
$$


hence

$$
\limsup _{n \rightarrow \infty} \frac{\sum_{j=1}^{n} a_{j} X_{j}}{b_{n}} \geq \limsup _{n \rightarrow \infty} \frac{a_{n} X_{n}}{b_{n}}=\infty \quad \text { almost surely }
$$

which completes the easy half of the proof.

From Theorem 1, we obtain a lower bound on the lower limit, i.e.,

$$
\liminf _{n \rightarrow \infty} \frac{\sum_{j=1}^{n} \frac{1}{j} X_{j}}{\lg ^{\alpha+1}(n) \lg _{2}(n)} \leq 1 \quad \text { almost surely. }
$$

In order to obtain equality here, we use the sequence $d_{n}$ and partition our sum as

$$
\begin{aligned}
\frac{1}{b_{n}} \sum_{j=1}^{n} a_{j} X_{j} \geq & \frac{1}{b_{n}} \sum_{j=1}^{n} a_{j} X_{j} I\left(0<X_{j} \leq d_{j}\right) \\
= & \frac{1}{b_{n}} \sum_{j=1}^{n} a_{j}\left[X_{j} I\left(0<X_{j} \leq d_{j}\right)-E X_{j} I\left(0<X_{j} \leq d_{j}\right)\right] \\
& +\frac{1}{b_{n}} \sum_{j=1}^{n} a_{j} E X_{j} I\left(0<X_{j} \leq d_{j}\right) .
\end{aligned}
$$

The first term converges to zero almost surely by the Khintchine-Kolmogorov Convergence Theorem, see [3], page 113 and the Kronecker Lemma since $b_{n} \uparrow \infty$ and

$$
\begin{aligned}
& \sum_{n=1}^{\infty} \frac{1}{c_{n}^{2}} E X_{n}^{2} I\left(0<X_{n} \leq d_{n}\right) \\
& =\sum_{n=1}^{\infty} \frac{1}{c_{n}^{2}} \int_{0}^{d_{n}} x^{2}\left(\frac{\lg ^{\alpha}(x+n)-\alpha \lg ^{\alpha-1}(x+n)}{(x+n)^{2}}\right) d x \\
& \leq \sum_{n=1}^{\infty} \frac{1}{c_{n}^{2}} \int_{n}^{d_{n}+n} \frac{(u-n)^{2} \lg ^{\alpha}(u) d u}{u^{2}} \\
& \leq \sum_{n=1}^{\infty} \frac{1}{c_{n}^{2}} \int_{n}^{d_{n}+n} \lg ^{\alpha}(u) d u \\
& \leq \sum_{n=1}^{\infty} \frac{\lg ^{\alpha}\left(d_{n}+n\right)}{c_{n}^{2}} \int_{n}^{d_{n}+n} d u \\
& \leq C \sum_{n=1}^{\infty} \frac{d_{n} \lg ^{\alpha}\left(d_{n}\right)}{c_{n}^{2}}
\end{aligned}
$$




$$
\begin{aligned}
& \leq C \sum_{n=1}^{\infty} \frac{n \lg ^{2 \alpha+1}(n)}{n^{2} \lg ^{2 \alpha+2}(n) \lg _{2}^{2}(n)} \\
& =C \sum_{n=1}^{\infty} \frac{1}{n \lg (n) \lg _{2}^{2}(n)}<\infty
\end{aligned}
$$

whenever $\alpha \geq 0$. If $-1<\alpha<0$, then

$$
\begin{aligned}
& \sum_{n=1}^{\infty} \frac{1}{c_{n}^{2}} E X_{n}^{2} I\left(0<X_{n} \leq d_{n}\right) \\
& =\sum_{n=1}^{\infty} \frac{1}{c_{n}^{2}} \int_{0}^{d_{n}} x^{2}\left(\frac{\lg ^{\alpha}(x+n)-\alpha \lg ^{\alpha-1}(x+n)}{(x+n)^{2}}\right) d x \\
& \leq C \sum_{n=1}^{\infty} \frac{1}{c_{n}^{2}} \int_{n}^{d_{n}+n} \frac{(u-n)^{2} \lg ^{\alpha}(u) d u}{u^{2}} \\
& \leq C \sum_{n=1}^{\infty} \frac{1}{c_{n}^{2}} \int_{n}^{d_{n}+n} \lg ^{\alpha}(u) d u \\
& \leq C \sum_{n=1}^{\infty} \frac{\lg ^{\alpha}(n)}{c_{n}^{2}} \int_{n}^{d_{n}+n} d u \\
& =C \sum_{n=1}^{\infty} \frac{d_{n} \lg ^{\alpha}(n)}{c_{n}^{2}} \\
& =C \sum_{n=1}^{\infty} \frac{n \lg ^{2 \alpha+1}(n)}{n^{2} \lg ^{2 \alpha+2}(n) \lg _{2}^{2}(n)} \\
& =C \sum_{n=1}^{\infty} \frac{1}{n \lg ^{2}(n) \lg _{2}^{2}(n)}<\infty .
\end{aligned}
$$

Next, we need to show that the other term converges to one as in the proof of Theorem 1, As with the expectation in Theorem 1, we have four terms.

The first term is the important one

$$
\begin{aligned}
& \frac{1}{b_{n}} \sum_{j=1}^{n} a_{j} E X_{j} I\left(0<X_{j} \leq d_{j}\right) \\
& =\frac{1}{b_{n}} \sum_{j=1}^{n} \frac{1}{j} \int_{0}^{d_{j}} x\left(\frac{\lg ^{\alpha}(x+j)-\alpha \lg ^{\alpha-1}(x+j)}{(x+j)^{2}}\right) d x \\
& =\frac{1}{b_{n}} \sum_{j=1}^{n} \frac{1}{j} \int_{j}^{d_{j}+j} \frac{(u-j)\left(\lg ^{\alpha}(u)-\alpha \lg ^{\alpha-1}(u)\right) d u}{u^{2}}
\end{aligned}
$$




$$
\begin{aligned}
= & \frac{1}{b_{n}} \sum_{j=1}^{n} \frac{1}{j} \int_{j}^{d_{j}+j} \frac{\lg ^{\alpha}(u) d u}{u}-\frac{\alpha}{b_{n}} \sum_{j=1}^{n} \frac{1}{j} \int_{j}^{d_{j}+j} \frac{\lg ^{\alpha-1}(u) d u}{u} \\
& -\frac{1}{b_{n}} \sum_{j=1}^{n} \int_{j}^{d_{j}+j} \frac{\lg ^{\alpha}(u) d u}{u^{2}}+\frac{\alpha}{b_{n}} \sum_{j=1}^{n} \int_{j}^{d_{j}+j} \frac{\lg ^{\alpha-1}(u) d u}{u^{2}} \\
= & I_{1}+I_{2}+I_{3}+I_{4} .
\end{aligned}
$$

The first term is

$$
\begin{aligned}
& \frac{1}{b_{n}} \sum_{j=1}^{n} \frac{1}{j} \int_{j}^{d_{j}+j} \frac{\lg ^{\alpha}(u) d u}{u}=\frac{1}{b_{n}} \sum_{j=1}^{n} \frac{1}{j} \int_{\lg (j)}^{\lg \left(d_{j}+j\right)} w^{\alpha} d w \\
& =\frac{1}{(\alpha+1) b_{n}} \sum_{j=1}^{n} \frac{1}{j}\left(\lg ^{\alpha+1}\left(d_{j}+j\right)-\lg ^{\alpha+1}(j)\right) \\
& =\frac{1}{(\alpha+1) b_{n}} \sum_{j=1}^{n} \frac{1}{j}\left(\left[\lg (j)+\lg \left(\lg ^{\alpha+1}(j)+1\right)\right]^{\alpha+1}-\lg ^{\alpha+1}(j)\right) \\
& =\frac{1}{(\alpha+1) b_{n}} \sum_{j=1}^{n} \frac{\lg ^{\alpha+1}(j)}{j}\left(\left[1+\frac{\lg \left(\lg ^{\alpha+1}(j)+1\right)}{\lg (j)}\right]^{\alpha+1}-1\right) .
\end{aligned}
$$

The only term of significance in $(1+x)^{\alpha+1}$ is the $(\alpha+1) x$ term, where $x=\lg \left(\lg ^{\alpha+1}(j)+1\right) / \lg (j)$, which is certainly smaller than one. Thus $I_{1}$

becomes

$$
\begin{aligned}
& \frac{1}{(\alpha+1) b_{n}} \sum_{j=1}^{n} \frac{\lg ^{\alpha+1}(j)}{j}\left(\frac{(\alpha+1) \lg \left(\lg ^{\alpha+1}(j)+1\right)}{\lg (j)}\right) \\
& =\frac{1}{b_{n}} \sum_{j=1}^{n} \frac{\lg ^{\alpha+1}(j)}{j}\left(\frac{\lg \left(\lg ^{\alpha+1}(j)+1\right)}{\lg (j)}\right) \\
& \sim \frac{1}{b_{n}} \sum_{j=1}^{n} \frac{\lg ^{\alpha+1}(j)}{j}\left(\frac{(\alpha+1) \lg _{2}(j)}{\lg (j)}\right) \\
& =\frac{\alpha+1}{b_{n}} \sum_{j=1}^{n} \frac{\lg ^{\alpha}(j) \lg _{2}(j)}{j} \\
& \sim\left(\frac{\alpha+1}{b_{n}}\right)\left(\frac{\lg ^{\alpha+1}(n) \lg _{2}(n)}{\alpha+1}\right)=1 .
\end{aligned}
$$

As for any other powers of $x$, those vanish as $n$ goes to infinity since they 
are smaller than

$$
\frac{C}{b_{n}} \sum_{j=1}^{n} \frac{\lg ^{\alpha+1}(j)}{j}\left(\frac{\lg \left(\lg ^{\alpha+1}(j)+1\right)}{\lg (j)}\right)^{p} \rightarrow 0
$$

for all $p \geq 2$. As for $I_{2}$ we have as an upper bound

$$
\left|\frac{-\alpha}{b_{n}} \sum_{j=1}^{n} \frac{1}{j} \int_{j}^{d_{j}+j} \frac{\lg ^{\alpha-1}(u) d u}{u}\right| \leq \frac{C}{b_{n}} \sum_{j=1}^{n} \frac{1}{j} \int_{\lg (j)}^{\lg \left(d_{j}+j\right)} w^{\alpha-1} d w .
$$

If $\alpha<0$ then this is less than

$$
\frac{C}{b_{n}} \sum_{j=1}^{n} \frac{1}{j} \int_{\lg (j)}^{\infty} w^{\alpha-1} d w \leq \frac{C}{b_{n}} \sum_{j=1}^{n} \frac{\lg ^{\alpha}(j)}{j} \leq \frac{C \lg ^{\alpha+1}(n)}{b_{n}} \leq \frac{C}{\lg _{2}(n)} \rightarrow 0 .
$$

If $\alpha=0$ then this term doesn't even exist and if $\alpha>0$, then this is bounded above by

$$
\frac{C}{b_{n}} \sum_{j=1}^{n} \frac{\lg ^{\alpha}\left(d_{j}+j\right)}{j} \leq \frac{C}{b_{n}} \sum_{j=1}^{n} \frac{\lg ^{\alpha}(j)}{j} \leq \frac{C \lg ^{\alpha+1}(n)}{b_{n}}=\frac{C}{\lg _{2}(n)} \rightarrow 0 .
$$

As for $I_{3}$ we have

$$
\begin{aligned}
\left|\frac{-1}{b_{n}} \sum_{j=1}^{n} \int_{j}^{d_{j}+j} \frac{\lg ^{\alpha}(u) d u}{u^{2}}\right| & \leq \frac{1}{b_{n}} \sum_{j=1}^{n} \int_{j}^{\infty} \frac{\lg ^{\alpha}(u) d u}{u^{2}} \\
& \leq \frac{C}{b_{n}} \sum_{j=1}^{n} \frac{\lg ^{\alpha}(j)}{j} \\
& \leq \frac{C \lg ^{\alpha+1}(n)}{b_{n}}=\frac{C}{\lg _{2}(n)} \rightarrow 0 .
\end{aligned}
$$

Finally, an upper bound for $I_{4}$ is

$$
\left|\frac{\alpha}{b_{n}} \sum_{j=1}^{n} \int_{j}^{d_{j}+j} \frac{\lg ^{\alpha-1}(u) d u}{u^{2}}\right| \leq \frac{C}{b_{n}} \sum_{j=1}^{n} \int_{j}^{\infty} \frac{\lg ^{\alpha-1}(u) d u}{u^{2}} \leq \frac{C}{b_{n}} \sum_{j=1}^{n} \frac{\lg ^{\alpha-1}(j)}{j} \rightarrow 0
$$

for any $\alpha$. Putting everything together we see that the lower limit is indeed one, with probability one.

We can also obtain a one sided strong law when $\alpha$ is minus one. 
Theorem 4. If $P\left\{X_{j}>x\right\}=\frac{1}{(x+j) \lg (x+j)}$, then

and

$$
\liminf _{n \rightarrow \infty} \frac{\sum_{j=1}^{n} \frac{1}{j} X_{j}}{\lg _{2}(n) \lg _{3}(n)}=1 \quad \text { almost surely }
$$

$$
\limsup _{n \rightarrow \infty} \frac{\sum_{j=1}^{n} \frac{1}{j} X_{j}}{\lg _{2}(n) \lg _{3}(n)}=\infty \quad \text { almost surely. }
$$

Proof. Let $a_{j}=1 / j, b_{n}=\lg _{2}(n) \lg _{3}(n), c_{n}=b_{n} / a_{n}=n \lg _{2}(n) \lg _{3}(n)$ and $d_{n}=n \lg _{2}(n)$. Once again, the difference between $c_{n}$ and $d_{n}$ is subtle, but that is exactly what helps us achieve our lower limit. The upper limit occurs since for all $M>0$

$$
\begin{aligned}
& \sum_{n=1}^{\infty} P\left\{a_{n} X_{n} / b_{n}>M\right\}=\sum_{n=1}^{\infty} P\left\{X_{n}>M c_{n}\right\} \\
& =\sum_{n=1}^{\infty} \frac{1}{\left(M c_{n}+n\right) \lg \left(M c_{n}+n\right)} \\
& =\sum_{n=1}^{\infty} \frac{1}{\left(M n \lg _{2}(n) \lg _{3}(n)+n\right) \lg \left(M n \lg _{2}(n) \lg _{3}(n)+n\right)} \\
& \geq C \sum_{n=1}^{\infty} \frac{1}{n \lg (n) \lg _{2}(n) \lg _{3}(n)}=\infty .
\end{aligned}
$$

Thus

$$
\limsup _{n \rightarrow \infty} \frac{a_{n} X_{n}}{b_{n}}=\infty \quad \text { almost surely }
$$

hence

$$
\limsup _{n \rightarrow \infty} \frac{\sum_{j=1}^{n} a_{j} X_{j}}{b_{n}} \geq \limsup _{n \rightarrow \infty} \frac{a_{n} X_{n}}{b_{n}}=\infty \quad \text { almost surely }
$$

which completes the easy half of the proof.

From Theorem 2, we obtain a lower bound on the lower limit, i.e.,

$$
\liminf _{n \rightarrow \infty} \frac{\sum_{j=1}^{n} \frac{1}{j} X_{j}}{\lg _{2}(n) \lg _{3}(n)} \leq 1 \quad \text { almost surely. }
$$


Using the same partition as in Theorem 3, but with a different $d_{n}$ we have

$$
\begin{aligned}
\frac{1}{b_{n}} \sum_{j=1}^{n} a_{j} X_{j} \geq & \frac{1}{b_{n}} \sum_{j=1}^{n} a_{j} X_{j} I\left(0<X_{j} \leq d_{j}\right) \\
= & \frac{1}{b_{n}} \sum_{j=1}^{n} a_{j}\left[X_{j} I\left(0<X_{j} \leq d_{j}\right)-E X_{j} I\left(0<X_{j} \leq d_{j}\right)\right] \\
& +\frac{1}{b_{n}} \sum_{j=1}^{n} a_{j} E X_{j} I\left(0<X_{j} \leq d_{j}\right)
\end{aligned}
$$

The first term converges to zero almost surely by the Khintchine-Kolmogorov Convergence Theorem, see [3], page 113 and the Kronecker Lemma since $b_{n} \uparrow \infty$ and

$$
\begin{aligned}
\sum_{n=1}^{\infty} \frac{1}{c_{n}^{2}} E X_{n}^{2} I\left(0<X_{n} \leq d_{n}\right) & =\sum_{n=1}^{\infty} \frac{1}{c_{n}^{2}} \int_{0}^{d_{n}} x^{2}\left(\frac{\lg (x+n)+1}{(x+n)^{2} \lg ^{2}(x+n)}\right) d x \\
& =\sum_{n=1}^{\infty} \frac{1}{c_{n}^{2}} \int_{n}^{d_{n}+n} \frac{(u-n)^{2}(\lg (u)+1) d u}{u^{2} \lg ^{2}(u)} \\
& \leq C \sum_{n=1}^{\infty} \frac{1}{c_{n}^{2}} \int_{n}^{d_{n}+n} \frac{d u}{\lg (u)} \\
& \leq C \sum_{n=1}^{\infty} \frac{1}{c_{n}^{2}} \int_{n}^{d_{n}+n} \frac{d u}{\lg (n)} \\
& =C \sum_{n=1}^{\infty} \frac{d_{n}}{c_{n}^{2} \lg (n)} \\
& =C \sum_{n=1}^{\infty} \frac{1}{n \lg (n) \lg _{2}(n) \lg _{3}^{2}(n)}<\infty
\end{aligned}
$$

Note that this barely converges and the previous infinite sum diverges, by the slimmest of margins, a factor of $\lg _{3}(n)$. Next, we need to show that the other term converges to one

$$
\begin{aligned}
& \frac{1}{b_{n}} \sum_{j=1}^{n} a_{j} E X_{j} I\left(0<X_{j} \leq d_{j}\right) \\
& =\frac{1}{b_{n}} \sum_{j=1}^{n} \frac{1}{j} \int_{0}^{d_{j}} x\left(\frac{\lg (x+j)+1}{(x+j)^{2} \lg ^{2}(x+j)}\right) d x
\end{aligned}
$$




$$
\begin{aligned}
= & \frac{1}{b_{n}} \sum_{j=1}^{n} \frac{1}{j} \int_{j}^{d_{j}+j} \frac{(u-j)(\lg (u)+1) d u}{u^{2} \lg ^{2}(u)} \\
= & \frac{1}{b_{n}} \sum_{j=1}^{n} \frac{1}{j} \int_{j}^{d_{j}+j} \frac{d u}{u \lg (u)}-\frac{1}{b_{n}} \sum_{j=1}^{n} \int_{j}^{d_{j}+j} \frac{d u}{u^{2} \lg (u)} \\
& +\frac{1}{b_{n}} \sum_{j=1}^{n} \frac{1}{j} \int_{j}^{d_{j}+j} \frac{d u}{u \lg ^{2}(u)}-\frac{1}{b_{n}} \sum_{j=1}^{n} \int_{j}^{d_{j}+j} \frac{d u}{u^{2} \lg ^{2}(u)} \\
= & I_{1}+I_{2}+I_{3}+I_{4} .
\end{aligned}
$$

The first integral is

$$
\begin{aligned}
\frac{1}{b_{n}} \sum_{j=1}^{n} \frac{1}{j} \int_{j}^{d_{j}+j} \frac{d u}{u \lg (u)} & =\frac{1}{b_{n}} \sum_{j=1}^{n} \frac{1}{j} \int_{\lg (j)}^{\lg \left(d_{j}+j\right)} \frac{d w}{w} \\
& =\frac{1}{b_{n}} \sum_{j=1}^{n} \frac{1}{j}\left[\lg _{2}\left(d_{j}+j\right)-\lg _{2}(j)\right] \\
& =\frac{1}{b_{n}} \sum_{j=1}^{n} \frac{1}{j} \lg \left(\frac{\lg \left(d_{j}+j\right)}{\lg (j)}\right) \\
& =\frac{1}{b_{n}} \sum_{j=1}^{n} \frac{1}{j} \lg \left(\frac{\lg (j)+\lg \left(\lg _{2}(j)+1\right)}{\lg (j)}\right) \\
& =\frac{1}{b_{n}} \sum_{j=1}^{n} \frac{1}{j} \lg \left(1+\frac{\lg (\lg (j)+1)}{\lg (j)}\right) .
\end{aligned}
$$

The only term of significance in $\lg (1+x)$ is $x=\lg \left(\lg _{2}(j)+1\right) / \lg (j)$. Thus

$I_{1}$ becomes

$$
\frac{1}{b_{n}} \sum_{j=1}^{n} \frac{1}{j}\left(\frac{\lg \left(\lg _{2}(j)+1\right)}{\lg (j)}\right) \sim \frac{1}{b_{n}} \sum_{j=1}^{n} \frac{1}{j}\left(\frac{\lg _{3}(j)}{\lg (j)}\right) \sim \frac{\lg _{2}(n) \lg _{3}(n)}{b_{n}}=1
$$

As for any other powers of $x$, those vanish as $n$ goes to infinity since they are smaller than

$$
\frac{1}{b_{n}} \sum_{j=1}^{n} \frac{1}{j}\left(\frac{\lg \left(\lg _{2}(j)+1\right)}{\lg (j)}\right)^{p} \leq \frac{1}{b_{n}} \sum_{j=1}^{n} \frac{\lg _{3}^{p}(j)}{j \lg ^{p}(j)} \leq \frac{C}{b_{n}} \rightarrow 0
$$


since $p \geq 2$. As for $I_{2}$ we have

$$
\begin{aligned}
\frac{1}{b_{n}} \sum_{j=1}^{n} \int_{j}^{d_{j}+j} \frac{d u}{u^{2} \lg u} & \leq \frac{1}{b_{n}} \sum_{j=1}^{n} \int_{j}^{\infty} \frac{d u}{u^{2} \lg u} \leq \frac{C}{b_{n}} \sum_{j=1}^{n} \frac{1}{j \lg j} \\
& \leq \frac{C \lg _{2}(n)}{b_{n}}=\frac{C}{\lg _{3}(n)} \rightarrow 0
\end{aligned}
$$

As for $I_{3}$ we have

$$
\begin{aligned}
\frac{1}{b_{n}} \sum_{j=1}^{n} \frac{1}{j} \int_{j}^{d_{j}+j} \frac{d u}{u \lg ^{2} u} & \leq \frac{1}{b_{n}} \sum_{j=1}^{n} \frac{1}{j} \int_{j}^{\infty} \frac{d u}{u \lg ^{2} u} \leq \frac{C}{b_{n}} \sum_{j=1}^{n} \frac{1}{j \lg j} \\
& \leq \frac{C \lg _{2} n}{b_{n}}=\frac{C}{\lg _{3} n} \rightarrow 0
\end{aligned}
$$

Finally for $I_{4}$ we have

$$
\frac{1}{b_{n}} \sum_{j=1}^{n} \int_{j}^{d_{j}+j} \frac{d u}{u^{2} \lg ^{2} u} \leq \frac{1}{b_{n}} \sum_{j=1}^{n} \int_{j}^{\infty} \frac{d u}{u^{2} \lg ^{2} u} \leq \frac{C}{b_{n}} \sum_{j=1}^{n} \frac{1}{j \lg ^{2} j} \leq \frac{C}{b_{n}} \rightarrow 0 .
$$

Putting everything together we see that the lower limit is once again one, with probability one.

\section{Discussion}

It shouldn't be too difficult to obtain similar Weak Laws and one-sided Strong Laws when $P\left\{X_{j}>x\right\}=\frac{\lg _{n}^{\alpha}(x+j)}{(x+j) \prod_{i=1}^{n-1} \lg _{i}(x+j)}$ whenever $\alpha \geq-1$ for any positive integer $n$. But to extend this to any sequence $m_{j}$ and any slowly varying function, $L(\cdot)$, where $P\left\{X_{j}>x\right\}=\frac{L\left(x+m_{j}\right)}{x+m_{j}}$ would be quite difficult.

\section{References}

1. A. Adler, Generalized one-sided laws of the iterated logarithm for random variables barely with or without finite mean, J. Theoret. Probab., 3 (1990), 587-597.

2. A. Adler, An exact weak law of large numbers, Bull. Inst. Math. Acad. Sinica, 7 (2012), 417-422.

3. Y. S. Chow and H. Teicher, Probability Theory: Independence, Interchangeability, Martingales, $3^{\text {rd }}$ ed., Springer-Verlag, New York, 1997.

4. W. Feller, An Introduction to Probability Theory and Its Applications, Vol 2, $2^{\text {nd }}$ ed., John Wiley, New York, 1966. 
5. T. Nakata, Weak law of large numbers for weighted independent random variables with infinite mean, Statist. Probab. Letters 109 (2016), 124-129. 